\title{
A dual rigidity of the sphere and the hyperbolic plane
}

\author{
Magdalena Caballero and Rafael M. Rubio \\ Departamento de Matemeticas, Campus de Rabanales, \\ Universidad de Córdoba, 14071 Córdoba, Spain, \\ E-mails: magdalena.caballero@uco.es, rmrubio@uco.es
}

\begin{abstract}
There are several well-known characterizations of the sphere as a regular surface in the Euclidean space. By means of a purely synthetic technique, we get a rigidity result for the sphere without any curvature conditions, nor completeness or compactness. As well as a dual result for the hyperbolic plane, the spacelike sphere in the Minkowski space.
\end{abstract}

2010 MSC: 53A05, 53A35, 53C24.

Keywords: Euclidean and Lorentzian Geometries, Sphere and Hyperbolic Plane.

\section{Introduction}

In 1897 Hadamard proved that any compact connected regular surface with positive Gaussian curvature in the three-dimensional Euclidean space $\mathbb{E}^{3}$ is a topological sphere, 3 . His result motivated the search for conditions to conclude that such a surface is necessarily a round sphere (an Euclidean sphere). Two answers were given by Liebmann. The first one, in 1899 [6], proved the rigidity of the sphere, conjectured by F. Minding in 1939,

If $S$ is a compact and connected regular surface in $\mathbb{E}^{3}$ with constant Gaussian curvature $K$, then $M$ is a sphere of radius $1 / \sqrt{K}$.

The second one involves the mean curvature, [7],

Any compact and connected regular surface in $\mathbb{E}^{3}$ with positive Gaussian curvature and constant mean curvature is a sphere.

Shortly after, Hilbert gave a simpler proof of the first result, [4]. His ideas where used by Chern in 2 to get a more general characterization of the sphere, concerning Weingarten surfaces. He obtained the previous two results by Liebmann as corollaries.

Another result on the sphere involving the Gaussian curvature, which is a direct consequence of a result by Hopf [5], asserts that it is the only complete and simply connected regular surface in $\mathbb{E}^{3}$ with positive constant Gaussian curvature.

The last characterization of the sphere we will mention is the Alexandrov theorem, which assures that a compact and connected regular surface of constant mean curvature in $\mathbb{E}^{3}$ is a sphere, [1]. 
Now consider the Minkowski space $\mathbb{L}^{3}$. A regular surface in this space is called spacelike if its induced metric is Riemannian. In this setting, the hyperbolic plane $\mathbb{H}^{2}$ can be realized as one connected component of the hyperboloid of two sheets, and so it can be viewed as the spacelike sphere in $\mathbb{L}^{3}$. Analogously to the (Euclidean) sphere, the hyperbolic plane can be characterized as the only spacelike regular surface in $\mathbb{L}^{3}$ which is complete, simply connected and with negative constant Gaussian curvature, [5].

In this work, we are interested in surfaces foliated by circles. R. López proved that $a$ surface in $\mathbb{E}^{3}$ with constant Gaussian curvature and foliated by pieces of circles is included in a sphere, or the planes containing the circles of the foliation are parallel, 8. In [9, the same author obtained the dual result in $\mathbb{L}^{3}$. It states that a spacelike surface in $\mathbb{L}^{3}$ with constant Gaussian curvature and foliated by pieces of circles must be a portion of a hyperbolic plane, unless the planes of the foliation are parallel.

This paper is devoted to prove natural dual characterizations of the sphere in $\mathbb{E}^{3}$ and the hyperbolic plane in $\mathbb{L}^{3}$. In our results neither the Gaussian curvature nor the mean curvature appear. Neither completeness nor compactness hypotheses are required. We only need a hypothesis on the intersection of the surfaces by planes.

More specifically, we say that a regular surface $S$ in the Euclidean space $\mathbb{E}^{3}$ satisfies the $\mathcal{P}$ property if for each affine plane $\Pi$ intersecting $S$, the set $\Pi \cap S$ is a circle (including the degenerate case with radius zero).

Analogously, we say that a spacelike regular surface $S$ in the Minkowski space $\mathbb{L}^{3}$ satisfies the $\mathcal{P}^{*}$ property if for each spacelike affine plane $\Pi$ intersecting $S$, the set $\Pi \cap S$ is a circle (including the degenerate case with radius zero). Notice that a circle in a spacelike affine plane $\Pi$ of $\mathbb{L}^{3}$ is the locus of the points in $\Pi$ at a constant distance from a fixed point in $\Pi$, where the distance considered is the one associated to the induced metric.

We state the following rigidity results:

Theorem 1.1 Let $S$ be a connected regular surface in the Euclidean space $\mathbb{E}^{3}$ satisfying the $\mathcal{P}$ property, then $S$ is necessarily an Euclidean sphere.

Theorem 1.2 Let $S$ be a spacelike connected regular surface in the Minkowski space $\mathbb{L}^{3}$ satisfying the $\mathcal{P}^{*}$ property, then $S$ is necessarily a hyperbolic plane.

\section{The proofs}

\section{Euclidean case.}

Let $S$ be a surface in $\mathbb{E}^{3}$ satisfying the $\mathcal{P}$ property and let $Q \in S$ be an arbitrary point. We consider the tangent plane $T_{Q} S$ and its normal line through $Q, \mathcal{L}$. We take the sheaf of affine planes with axis $\mathcal{L}$ and we denote by $\left\{C_{i}\right\}_{i \in I}$ the family of circles obtained when intersecting those planes with $S$.

We consider a plane $\Pi_{0}$ parallel to $T_{Q} S$ such that $C=\Pi_{0} \cap S$ is a non degenerate circle and we denote $P=\Pi_{0} \cap \mathcal{L}$. Then $\Pi_{0} \cap C_{i} \neq \emptyset$ for all $i \in I$, and the intersection points of each circle $C_{i}$ with $\Pi_{0}$ are the opposite points of a chord of $C_{i}$ contained in $\Pi_{0}$ with midpoint $P$. Therefore, the point $P$ must be the center of $C$ and as direct consequence the circles $C_{i}$ have all the same radius. Thus, the sphere given by $\bigcup_{i \in I} C_{i}$ is contained in $S$. We finish the proof thanks to the connectedness of $S$.

\section{Lorentzian case.}


Let $S$ be a surface in $\mathbb{L}^{3}$ satisfying the $\mathcal{P}^{*}$ property and let $\Pi_{0}$ be a spacelike plane such that $C=\Pi_{0} \cap S$ is a non-degenerate circle. We denote its center by $P$ and the normal line through $P$ by $\mathcal{L}$.

Firstly, we prove that $\mathcal{L} \cap S \neq \emptyset$. We proceed by contradiction. Let us assume $\mathcal{L} \cap S=\emptyset$. Therefore, any plane parallel to $\Pi_{0}$ either does not intersect $S$ or it does it in a non-degenerate circle whose interior contains a point of $\mathcal{L}$. We deduce that any line parallel to $\mathcal{L}$ intersects $S$ at most in one point, otherwise the intersection of $S$ and the plane generated by both lines contains a non-spacelike curve. Thus, we have proved that $S$ is a graph over a domain of $\Pi_{0}$ not intersecting $\mathcal{L}$ and foliated by circles. Since $S$ is spacelike, it can not be asymptotic to $\mathcal{L}$ or any line parallel to it, and so $\partial S \neq \emptyset$. If $\partial S$ does not contain a point of $\mathcal{L}$, then it contains a circle. In both cases we can find spacelike planes intersecting $S$ in a non closed curve, which is a contradiction.

We notice that $S$ must by closed, otherwise we proceed as before to arrive to a contradiction. We take a point $Q$ at which the distance from $P$ to $\mathcal{L} \cap S$ is attained.

For each $A \in C$ and for each chord perpendicular to the segment $A P$, we call its midpoint $A_{m}$. We can chose the chord as close to $A$ as necessary so that the plane generated by it and the segment $A_{m} Q$ is spacelike, we denote it by $\Pi_{A_{m}}$. We define $\varepsilon_{A}$ to be the supremum (in the set of all possible chords satisfying the previous property) of the distance from $A$ to $A_{m}$.

If $\varepsilon=\min _{C} \varepsilon_{A}$, we take $0<\rho<\varepsilon$ and for each $A \in C$ we consider the chord with $d\left(A_{m}, A\right)=\rho$. Hence, all the circles $\Pi_{A_{m}} \cap S$ have the same radius, and so there is a hyperbolic cap contained in $S$ and containing $C$.

Finally, for each point $A \in S$ there exists a spacelike plane intersecting $S$ in a nondegenerate circle containing $A$. Therefore, there exists a hyperbolic cap contained in $S$ and containing $A$. We finish the proof by using a connectedness argument.

\section{Acknowledgments}

The authors are partially supported by the Spanish MICINN Grant with FEDER funds MTM2010-18099.

\section{References}

[1] A. Alexandrov, Uniqueness theorems for surfaces in the large, Vestnik Leningrad Univ., $13(1958) 5-8$.

[2] S. S. Chern, Some new characterizations of the Euclidean sphere, Duke Math. J., 12 (1945), 270-290.

[3] J. Hadamard, Sur certaines propriétés des trajectoires en dynamique, J.Math.Pures Appl. 3 (1897), 331-387.

[4] D. Hilbert, Grundlagen der geometrie, 3rd ed., Leipzig, 1909.

[5] H. Hopf, Zum Clifford-Kleinschen Raumproblem, Math. Ann. 95 (1926), 313-339.

[6] H. Liebmann, Eine neue eigenschaft der kugel, Nachr. Kgl. Ges. Wiss. Göttingen, Math.Phys. Klasse (1899), 44-55. 
[7] H. Liebmann, Über die Verbiegung der feschlossenen Flächen positiver Krümmung, Math. Ann. 53 (1900), 81-112.

[8] R. López, Surfaces of constant Gauss curvature, Houston Journal of Mathematics, 27 (2001), 799-805.

[9] R. López, Surfaces of constant Gauss curvature in Lorentz-Minkowski Tree-space, Rocky Mountain Journal of Mathematics, 33 (2003), 971-993. 